\title{
Editorial
}

\section{International Bull Fertility Conference - Theory to Practice, Westport, Ireland, 2018}

\author{
Michael G Diskin ${ }^{1 \dagger}$, Pat Lonergan ${ }^{2 \dagger}$, David A. Kenny ${ }^{3 \dagger}$ and Sean Fair ${ }^{4 \dagger}$ \\ ${ }^{1}$ Teagasc, Teagasc, Animal \& Grassland Research and Innovation Centre, Athenry, Co. Galway. Ireland; ${ }^{2}$ School of Agriculture and Food Science, University College \\ Dublin, Dublin, Ireland; ${ }^{3}$ Teagasc, Animal and Grassland Research and Innovation Centre, Grange, Dunsany, Co. Meath, Ireland; ${ }^{4}$ Department of Biological Sciences, \\ Faculty of Science and Engineering, University of Limerick, Ireland
}

This supplement to Animal contains the papers associated with the keynote lectures delivered at the International Bull Fertility Conference - Theory to Practice held in Westport, Ireland from May 27th to 30th 2018. The conference followed on from the hugely successful International Conference on Cow Fertility held at the same venue in May 2014, the proceedings of which were published in Animal, Vol 8 Issue S1 May 2014 (see http://bit.ly/2sT6a8X). The conference was organised under the auspices of the British Society of Animal Science (BSAS) in close collaboration with Teagasc, University College Dublin, University of Limerick, the Cattle Association of Veterinary Ireland (CAVI), XL Vets, the British Cattle Veterinary Association (BCVA) and the Department of Agriculture Food and the Marine.

Fertility of the bull is a major contributor to overall reproductive performance, particularly in herds where natural service is predominately used. While a majority of dairy bred calves are sired through artificial insemination (Al), in excess of $80 \%$ of calves born to beef cows are typically sired by natural service stock bulls. In such situations, particularly where a high cow to bull ratio predominates or in small herds, having only one service sire. The fertility of the stock bull is of major importance. There is significant variation in fertility amongst individual bulls; while the reported incidence of sterility is generally low $(<4 \%)$, subfertility, at a consistent level of $20-25 \%$, is much more common in breeding bulls. Subfertility may be caused directly by low libido, sperm defects or indirectly by physical factors affecting bull mobility or mating ability. While a sub-fertile bull is capable of getting some cows pregnant it will result in low herd pregnancy rates, an extended calving interval, reduced calf weaning weights and higher involuntary culling of cows, unless the bull is operating within a herd with a very low cow:bull ratio. Frequently, subfertile bulls go undetected and

${ }^{\dagger}$ E-mail: michael,diskin@teagasc.ie; pat.lonergan@ucd.ie; david.kenny@ teagasc.ie; sean.fair@ul.ie the suspicion of subfertility does not become apparent until late in the breeding season or until such time that cows are checked for pregnancy. For several decades, the preponderance of research effort in the field of bovine reproductive physiology has focused on ways to improve cow fertility. Less emphasis has been placed on male fertility and few conferences specifically dedicated to the subject of bull fertility have taken place. Thus, the influence of the bull on herd fertility in both dairy and beef herds has largely been overlooked with the predominant focus being on cow infertility. While the exact proportion of poor herd reproductive efficiency that can be explained by the fertility of an individual bull is difficult to establish, given that semen from elite bulls can be used simultaneously in several countries, often reaching $>100,000$ inseminations per year, the fertility of an individual bull can have a major impact on productivity and economic returns. This is particularly important in seasonal pasture-based systems where high 6-week in-calf rates are of paramount importance. The overall aim of this conference was to fill the gap by aspiring to be a 'one-stop shop' for academics, industry professionals and veterinary practitioners with an interest in bull fertility. The breath of the invited papers in this supplement extends from understanding the basic cellular regulation of reproductive processes to cutting edge technologies and the field application of this knowledge - hence the sub-title Theory to Practice. Leading experts from around the globe gathered to discuss the significant developments and challenges facing bull fertility, from the genomic revolution in dairy cattle breeding to reliably predicting the fertility of individual bulls in the laboratory. Specialists from academia, veterinary practice and industry discussed the latest developments in male reproductive physiology, nutrition, sexual maturation, genomics of bull fertility and developments in the application of sex-sorted semen.

The sequence of events leading to the commercial application of Al date back to the discovery of sperm in 1678, although it took almost 100 years to demonstrate that sperm 
were the agents of fertilization and a further 100 years for the detailed events of fertilization to be elucidated. It was not until the early to mid-1900s that practical methods for Al were successfully developed, first in Russia and then in Denmark, where the first Al cooperative was established in 1936, followed by others in the US (Lonergan, 2018).

The critical events in development of the male reproductive system in cattle occur during the first 3 to 4 months of gestation and the first 6 to 9 months post natally (McGowan et al., 2018). The hypothalamic-pituitary-gonadal axis matures earlier in the bovine fetus than other domestic species, with descent of the testes into the scrotum occurring around the fourth month of gestation. The testis have both an exocrine function, involving the production of spermatozoa, release into the lumen of the seminiferous tubules and storage in the epididymis, and an endocrine function performed by the Leydig cells, the main function of which is the production of steroids. Spermatogenesis is a finely regulated process of germ cell multiplication and differentiation leading to the production of spermatozoa in the seminiferous tubules. In bulls, the total duration of spermatogenesis is 61 days, i.e. 4.5 times the duration of the cycle of the seminiferous epithelium (Staub and Johnston, 2018).

To optimize reproductive function for either natural service or Al, bulls should produce large numbers of morphologically normal fertile sperm. Therefore, knowledge regarding factors controlling gonadal development and puberty, the importance of testis size in breeding soundness, and mechanisms regarding testicular thermoregulation is of great importance (Kastelic, 2018). The advent of genomic selection (Taylor et al., 2018) has led to increased interest within the cattle breeding industry to market semen from young bulls as early as possible. Recent evidence suggests that an enhanced plane of nutrition in early life leads to an advancement of testicular development and the age at which puberty is attained (Kenny and Byrne, 2018). Such information on how nutrition mediates the interaction between the neuroendocrine system and testicular function will facilitate optimization of nutrition strategies to optimise sexual maturation and subsequent semen production in bulls.

Artificial insemination is the single most important technique devised to facilitate the genetic improvement of livestock. Artificial insemination has played a key role in eliminating diseases, facilitating global dissemination of superior genetics and particularly accelerating genetic gain at industry level. A bewildering variety of reproductive programmes using natural service, estrus synchronization prior to timed natural service or timed Al, as well as rapid resynchronization protocols for animals not conceiving to a particular insemination, have been developed (Baruselli et al., 2018). Such technologies contribute to improved livestock production efficiency and, consequently, improved profitability. The ability to predict a bull's fertility before semen is released into the field has been a long term objective of the animal breeding industry; the recent shift in the dairy industry towards the intensive use of young genomicallyselected bulls has increased its urgency. The technology available to assess semen characteristics has advanced greatly in recent years, including computer-assisted sperm analysis and flow-cytometric assessment of a variety of quality-related parameters (Harstine et al., 2018). The Al industry utilises many of these technologies to make decisions on whether to release for sale or discard a product. While no single in vitro bioassay of sperm quality has been successful in predicting sire fertility, correlations have been reported. Understanding the underlying causes of variation in bull fertility is a key prerequisite to achieving the goal of developing such a sperm bioassay (Fair and Lonergan, 2018) and must be coupled with knowledge of the physiological charateristics associated with semen processing as well as bull behaviour and libido and proper management during semen collection to maximize output per bull (Schenk, 2018).

The objective of an insemination is to ensure that there is an adequate reservoir of competent, capacitated, motile sperm in the caudal region of the oviductal isthmus, the site of the main sperm reservoir in the cow, at the time of ovulation to ensure fertilisation. Handling of semen straws, site of semen placement and timing of insemination relative to oestrus and ovulation are all critical factors that can impact on the pregnancy rate (Diskin, 2018). The role of seminal plasma deposited during natural mating in pregnancy establishment has been the subject of some debate. Data from rodents suggests that it is crucial in setting up an immunologically receptive environment for subsequent embryo development. However, embryo transfer studies in cattle would suggest that it is, at least, not essential for pregnancy, although it may induce an immune response in the female reproductive tract to facilitate sperm transport and the early stages of embryo development (Bromfield, 2018). Sperm interaction with the luminal fluid and epithelia of the female reproductive tract after deposition has a profound influence on pregnancy rates. An understanding of how these processes are coordinated can impact on improved semen storage, prolonged sperm lifespan in vivo and, ultimately, improved fertility (Miller, 2018). Once they reach the site of fertilisation in the oviduct, interaction between the oocyte and the fertilising sperm involves a complex cascade of events that ultimately lead to the union of the paternal and maternal genomes in the zygote prior to the first mitotic cleavage division (Sutovsky, 2018). Sexed semen is now a commercial reality. Next year will mark 30 years since the first report of live offspring following sex-sorting of sperm. Comparable fertility of sex-sorted semen with conventional semen has been an aspirational benchmark for the industry for many years and the gap between the two is narrowing with refinements in the technology (Vishwanath and Moreno, 2018). It is important to note that, particularly in seasonal systems of production, even a small decrease in fertility relative to conventional semen can negate much of the economic benefit associated with sex-sorted semen (Holden and Butler, 2018).

Because of the serious implications of an infertile or subfertile bull on herd productivity, carrying out a Bull Breeding Soundness Evaluation (BBSE) pre-breeding is increasingly seen as a good "insurance policy" to reduce the risk of a 
subfertile bull. This evaluation is a useful, low-cost screening method for reducing the risk of low fertility bulls (Barth, 2018). Selecting bulls for reproductive soundness requires that the bull be structurally sound, free of abnormalities that impair his ability to produce adequate numbers of motile, morphologically normal spermatozoa, and be able to successfully complete coitus. The diagnosis and etiology of abnormalities of the penis, prepuce as well as common musculoskeletal conditions that limit normal breeding soundness are critical (Wolfe, 2018).

Lastly, knowledge concerning pathogens that could potentially be transmitted via bovine semen is crucial to achieving high reproductive rates. As a result of a careful analysis of the characteristics of infections that may cause transmission of disease through semen, effective control procedures can be identified that provide minimal constraint to the introduction of new bulls into herds for natural breeding and importation of valuable novel genetics through artificial insemination (Givens, 2018).

\section{Conclusions}

In conclusion, we hope that the papers in this supplementary issue of Animal will serve to highlight the importance of the topic of bull fertility and the unique and comprehensive nature of the conference in tackling this complex and multi-faceted subject.

\section{Acknowledgements}

A special word of thanks is due to the Scientific Committee who generously gave of their time in organising this Conference, including reviewing the papers in this Special Issue of Animal, chairing the scientific sessions and reviewing the abstracts. Sincere thanks are also due to staff at BSAS, particularly Bruce Beveridge, Bridget Hilton, Leigh Murray and Jon Day for their enthusiasm and help in organisation at all levels and to the staff at Animal for their patience and care in the production of this unique and comprehensive publication.

\section{References}

Barth AD 2018. The use of bull breeding soundness evaluation to identify subfertile and infertile bulls. Animal 12 (S1), s158-164.

Baruselli PS, Ferreira RM, Sá Filho MF and Bó GA 2018. Using artificial insemination v. natural service in beef herds. Animal 12 (S1), s45-52.

Bromfield JJ 2018. The potential of seminal fluid mediated paternal-maternal communication to optimise pregnancy success. Animal 12 (S1), s104-109.

Diskin MG 2018. Semen handling, time of insemination and insemination technique in cattle. Animal 12 (S1), s75-84.

Fair $S$ and Lonergan $P$ 2018. Understanding the causes of variation in reproductive wastage among bulls. Animal 12 (S1), s53-62.

Givens MD 2018. Risks of disease transmission through semen in cattle. Animal 12 (S1), s165-171.

Harstine BR, Utt MD and DeJarnette JM 2018. Integrating a semen quality control program and sire fertility at a large artificial insemination organization. Animal 12 (S1), s63-74.

Holden SA and Butler ST 2018. Applications and benefits of sexed semen in dairy and beef herds. Animal 12 (S1), s97-103.

Kenny DA and Byrne CJ 2018. The effect of nutrition on timing of pubertal onset and subsequent fertility in the bull. Animal 12 (S1), s36-44.

Kastelic JP 2018. Testicular vascular cone development and its association with scrotal thermoregulation, semen quality and sperm production in bulls. Animal 12 (S1), s133-141.

Lonergan P 2018. Historical and futuristic developments in bovine semen technology. Animal 12 (S1), s4-18.

McGowan M, Holland MK and Boe-Hansen G 2018. Ontology and endocrinology of the reproductive system of bulls from fetus to maturity. Animal 12 (S1), s19-26.

Miller DJ 2018. The epic journey of sperm through the female reproductive tract. Animal 12 (S1), s110-120.

Schenk JL 2018. Principles of maximizing bull semen production at genetic centers. Animal 12 (S1), s142-147.

Staub $C$ and Johnson $L$ 2018. A review of spermatogenesis in the bull. Animal 12 (S1), s27-35.

Sutovsky P 2018. Sperm-oocyte interactions and their implications for bull fertility, with emphasis on the ubiquitin-proteasome system. Animal 12 (S1), s121-132.

Taylor JF, Schnabel RD and Sutovsky P 2018. Genomics of bull fertility. Animal 12 (S1), s172-183.

Vishwanath R and Moreno JF 2018. Semen sexing - current state of the art with emphasis on bovine species. Animal 12 (S1), s85-96.

Wolfe DF 2018. Abnormalities of the bull - occurrence, diagnosis and treatment of abnormalities of the bull, including structural soundness. Animal 12 (S1), s148-157. 